\title{
Design of Low Cost Microstrip Antenna Arrays for mm-Wave Applications
}

\author{
Amin Rida and Manos Tentzeris \\ Electrical Engineering department \\ Georgia Institute of Technology \\ Atlanta, GA, USA \\ arida@ece.gatech.edu
}

\author{
Symeon (Simos) Nikolaou \\ Frederick Research Center and Frederick University \\ Nicosia, Cyprus
}

\section{SUB-ARRAY ANTENNA DESIGN}

\begin{abstract}
A planar, low profile, low cost microstrip patch array antenna is developed in the millimeter-wave band for large gain applications. A simple edge feeding is used for a sub-array of 16x1 elements, and finally a matching network or power divider/splitter is designed for a $32 \times 16$ elements with a gain of $27 \mathrm{dBi}$ for the design frequency of $79 \mathrm{GHz}$ and $26 \mathrm{dBi}$ at $80 \mathrm{GHz}$. A method to reduce the slightly high "shoulder" radiation due to the feed network is introduced through a shielded feed.
\end{abstract}

Index Terms: millimeter-wave, microstrip antenna, array antenna.

\section{INTRODUCTION}

The advantage of mm-Wave frequencies is better resolution and higher data rates if compared with applications in the lower frequency spectrum.; both which are necessary for systems with better capabilities and higher levels of functionality such as broadband and Gigabit wireless networks, vehicular radars, remote sensing of clouds and terrain, as well as imaging systems. Millimeter-wave antennas are being developed for several of these applications and research has been focused on the realization of mm-Wave hardware [1]. It is the result of the enabling technologies such as MMICs developed using SiGe and GaAs up till frequencies above $100 \mathrm{GHz}$ as well as CMOS around $60 \mathrm{GHz}$ that created this need for antennas and packaging considerations for these frequencies [2].

The focus to date therefore is directed towards successful hardware demonstration that fulfils required functionalities for mm-Wave technologies such as high gain, low profile, and low cost or the potential for low manufacturing costs. The work in this paper is targeted towards a simple design for a large antenna array characterized by a low profile for $79 \mathrm{GHz}$ and $80 \mathrm{GHz}$ frequencies [3] for the mm-Wave UWB applications such as Short Range Radar (SRR) for vehicles. The structure is designed and simulated in the 3D electromagnetic simulator CST Microwave Studio and utilizes the parameters of commercially available substrate RO3003.

\section{A. Sub-Array Design 16x1}

A 16x1 sub-array of individual patch antennas of size $1.2 \mathrm{~mm} \times 1 \mathrm{~mm}$ is designed in this section. The size of the individual element was chosen for the successful operation of the sub-array at the two frequencies $79 \mathrm{GHz}$ and $80 \mathrm{GHz}$. The element spacing was chose to have a spacing that is close to $\lambda_{\mathrm{g}} / 2$ or equivalently to create an in phase excitation among all of the elements at the given design frequencies. The layout of the design and its dimensions are shown in Figure 1. This subarray is designed on a 5 mil thick $(0.127 \mathrm{~mm})$ RO3003 material with dielectric constant $\left(\varepsilon_{\mathrm{r}}\right) 3.0$ and dielectric loss tangent $(\tan \delta)$ 0.0013. It is also to be noted that the metallization was modeled as copper with a thickness of $9 \mu \mathrm{m}$; a common metallization thickness for mm-Wave circuitry [4].

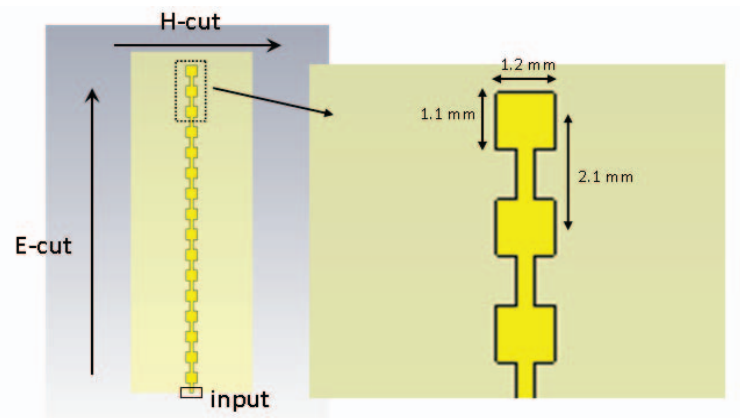

Figure 1. Layout of the 16x1 Sub-array.

\section{B. S-parameter and gain simulation results.}

The S-parameter results for the $16 \times 1$ sub-array is shown in Figure 2 demonstrating a very good matching for the design frequencies. Figure 3 shows the gain pattern for the Ecut (as defined in Figure 1). In the following figures theta resembles the elevation plane and phi resembles the azimuth plane. The pattern shows a maximum gain of $17.8 \mathrm{dBi}, 3 \mathrm{~dB}$ angular width of $5.6^{\circ}$, and a side-lobe level (SLL) $-10.7 \mathrm{~dB}$. For sake of space limitation the rest of the paper will focus on the $80 \mathrm{GHz}$ frequency but it is to be noted that the $79 \mathrm{GHz}$ has equivalently good performance. Figure 4 shows the gain pattern for the E-cut at $80 \mathrm{GHz}$ with a maximum gain of 
$17.9 \mathrm{dBi}, 3 \mathrm{~dB}$ angular width of $5.8^{\circ}$, and a side-lobe level (SLL) $-12.1 \mathrm{~dB}$. Figure 5 shows the $\mathrm{H}$-cut $(\mathrm{Phi}=0)$ of the $16 \mathrm{x} 1$ sub-array at $80 \mathrm{GHz}$ with a $3 \mathrm{~dB}$ angular width of $75^{\circ}$.

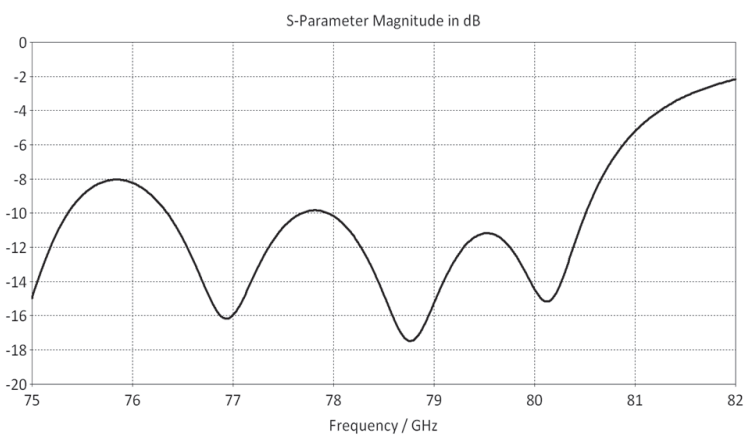

Figure 2. $\mathrm{S}_{11}$ for the $16 \times 1$ Sub-array shown in Figure 1.

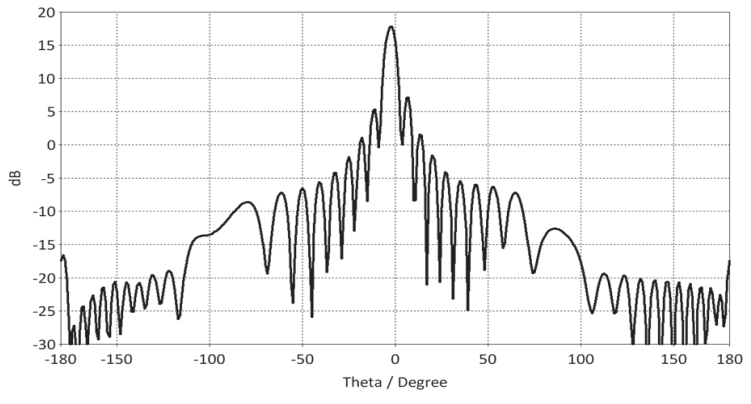

Figure 3. Gain (E-cut, Phi=90) for the $16 \times 1$ Sub-array at $79 \mathrm{GHz}$.

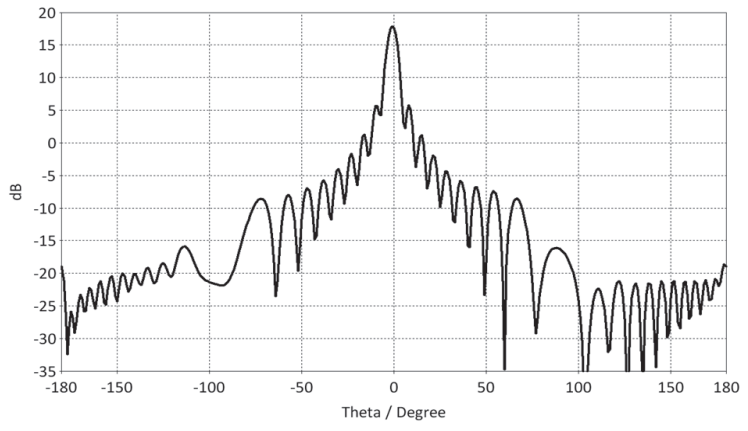

Figure 4. Gain (E-cut, Phi=90) for the $16 \times 1$ Sub-array at $80 \mathrm{GHz}$.

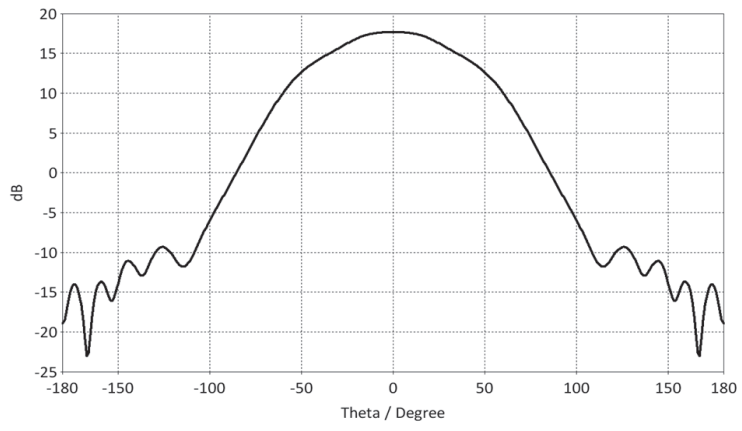

Figure 5. Gain (H-cut, Phi=0) for the $16 \times 1$ Sub-array at $80 \mathrm{GHz}$.

\section{LARGE ANTENNA ARRAY DESIGN}

An ideal phased array antenna may utilize phase shifters at the input of each channel or sub-array; something which is also ideal for lower loss performance. An ideal $16 \times 32$ array with center to center spacing of $1.9 \mathrm{~mm}$ of adjacent sub-arrays was simulated for a total gain of $31.5 \mathrm{dBi}$ at $80 \mathrm{GHz}$ and a SLL of $-12 \mathrm{~dB}$. The next section talks about the same $16 \times 32$ array but with a feeding network in order to have a 1-Port device for measurement purposes.

\section{A. Array Design 16x32}

A corporate feed was designed using quarter wavelength sections at the design frequencies and tapered corners to eliminate any undesired radiation due to sharp edges at such high frequencies. The design is shown in Figure 6.

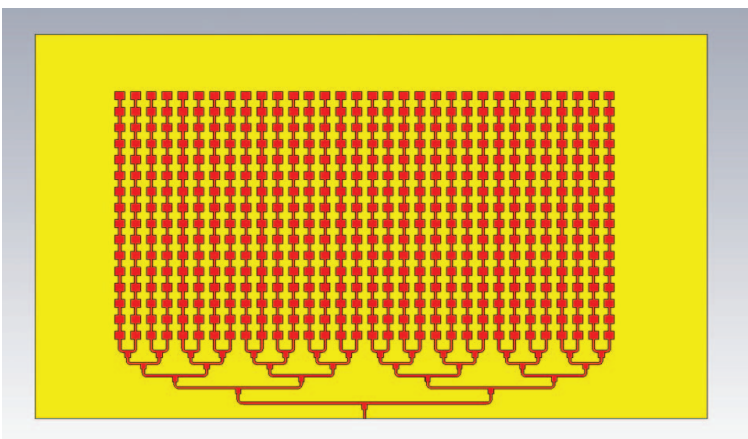

Figure 6. Layout of the $16 \times 32$ Array with feeding network.

\section{B. S-parameter and gain simulation results.}

The S-parameter results for the $16 \times 32$ array including the corporate feeding network is shown in Figure 7 demonstrating a very good matching for the design frequencies. The $S_{11}$ at $79 \mathrm{GHz}$ is $-13 \mathrm{~dB}$ and at $80 \mathrm{GHz}$ is $-12 \mathrm{~dB}$. Figure 8 shows the gain pattern (E-cut) of the array of Figure 6 with a maximum gain of $26.2 \mathrm{dBi}$, a $3 \mathrm{~dB}$ angular width of $6.1^{\circ}$, and a SLL of $11.1 \mathrm{~dB}$ and a radiation efficiency of $78 \%$. An observation worth mentioning here is the increased level of the side lobes due to the feeding network as its dimensions starts to affect the radiation performance in the $\mathrm{mm}$-Wave frequencies. 


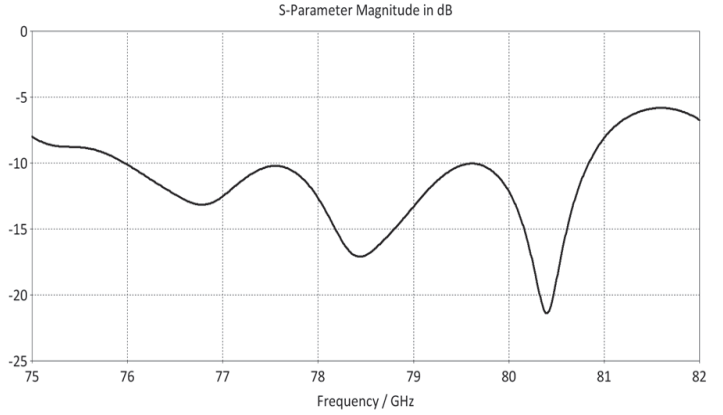

Figure 7. $S_{11}$ for the $16 \times 32$ Sub-array shown in Figure 6 .

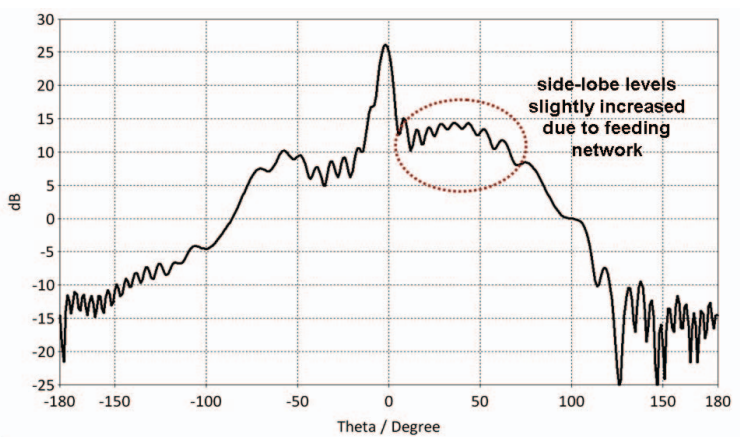

Figure 8. Gain (E-cut, Phi=90) for the 16x32 Antenna Array at $80 \mathrm{GHz}$.

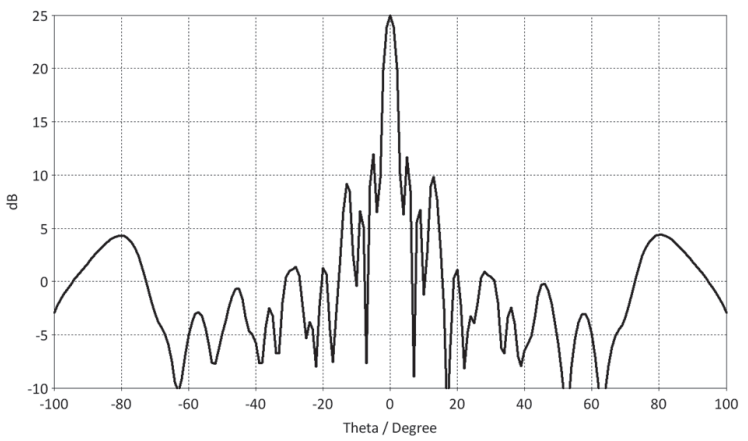

Figure 9. Gain (H-cut, Phi=0) for the $16 \times 32$ Antenna Array at $80 \mathrm{GHz}$.

The H-cut gain plot is also shown in Figure 9 resembling the array factor for 32 sub-arrays or elements with spacing (center to center) of $1.9 \mathrm{~mm}$ having a $3 \mathrm{~dB}$ angular width of $3^{\circ}$ and a SLL of $-14 \mathrm{~dB}$.

\section{IV. “SHOULDER” RADIATION REDUCTION DUE TO CORPORATE FEEDING}

In order to reduce the effect of the feeding network RF 3D interconnections may be utilized [4] or simply a shielded corporate feeding such as the one shown in Figure 10 which includes an unsymmetrical strip line configuration for the corporate feeding (15 mils substrate above the microstrip line, 5 mils below it). The gain pattern in Figure 10 has a major shoulder radiation improvement when compared to that shown in Figure 8 and a slight increase in the maximum gain from $26.2 \mathrm{dBi}$ to $26.5 \mathrm{dBi}$.

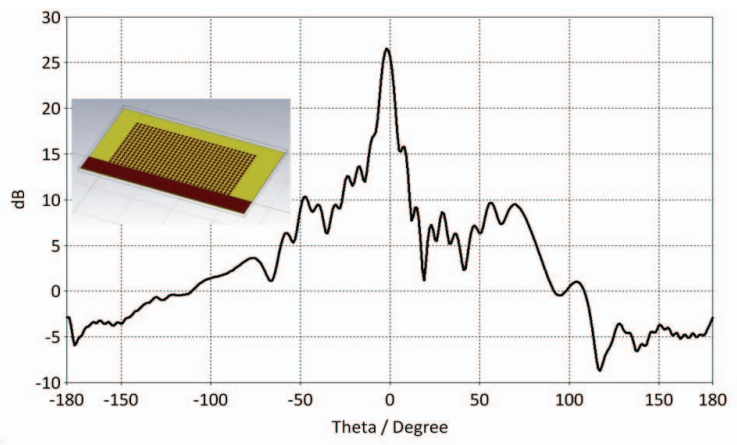

Figure 10. Gain (E-cut, Phi=90) for the $16 \times 32$ Antenna Array at $80 \mathrm{GHz}$

\section{CONCLUSION AND FUTURE WORK}

A planar, low profile, low cost microstrip patch array antenna has been developed for the millimeter-wave band. A simple edge feeding has been used for a sub-array of $16 \times 1$ elements, and a matching network was designed for a 32x16 elements with a gain of $27 \mathrm{dBi}$ for the design frequency of $79 \mathrm{GHz}$ and $26 \mathrm{dBi}$ at $80 \mathrm{GHz}$ with a radiation efficiency of $78 \%$. A method to reduce the slightly high "shoulder" radiation due to the feed network has been introduced through an unsymmetric strip line configuration feeding. Future work includes the experimental characterization of the work mentioned in this paper and the possibility of a lower loss feeding network.

\section{REFERENCES}

[1] K.C. HuANG AND D.J. EDWARDS, Millimeter Wave Antennas for Gigabit Wireless Communications, WILEY, 2008

[2] A. EZZEDINE, "Advances in Microwave and Millimeter-wave Integrated Circuits" in National Radio Science Conference, pp. 1-8, NRSC March 2007

[3] D. Platt, L. Pettersson, D. Jakonis, M. Salter, And J. HagGlunel, "Integrated 79GHz UWB Automotive Radar Front-End based on HiMission MCM-D Silicon Platform" in the Proceedings of the $6^{\text {th }}$ European Radar Conference, October 2009, Rome, Italy

[4] A. Rida, A. Margomenos, J. LeE, P, Schmalenberg, S. Nikolaou, AND M.M. TENTZERIS, "Integrated Wideband 2D \& 3D Transitions for Millimeter-Wave RF Front-Ends" IEEE Antennas and Wireless Propagation Letters, Vol. 9, pp. 1080-1083, 2010 